\title{
Influenza vaccine safe and effective in immunosuppressed patients
}

Patients with IBD should receive annual vaccination against influenza, regardless of whether they receive immunosuppressive therapy, report $\mathrm{Lu}$ and colleagues in their open-label, prospective study.

Immunosuppressive therapy is often prescribed for patients with IBD. While such regimens may improve symptoms of IBD, they also place patients at increased risk for development of diseases that are preventable by vaccination. Annual vaccination against common infections such as influenza is, therefore, recommended in this group of patients. Despite these recommendations, little is known about the effects of vaccines in patients with IBD. A study performed at the Children's Hospital Boston, MA, aimed to address several major, unanswered questions: do IBD patients on immunosuppressive medication respond normally to vaccines, do these patients develop protective levels of antibody, are vaccines safe, and can they trigger flares of the underlying disease?

\section{4 ....children developed} protective levels of antibody ... irrespective of [their] immunosuppressive therapy... 77

The researchers measured influenza antibody titer in the blood of 146 children with IBD before administration of an influenza vaccine, which contained proteins from three strains of influenza virus. The children returned 3-9 weeks after the vaccine had been administered and their antibody titers were measured again. Results were categorized according to whether the children were receiving immunosuppressive therapy. "The most significant finding was that children developed protective levels of antibody to the vaccine, irrespective of what immunosuppressive therapy they were receiving," explains the lead researcher, Athos Bousvaros. "We also did not see any increase in adverse events or increase in disease flares," he continues.

The findings from this study demonstrate the effectiveness and safety of the influenza vaccine in patients with IBD who are on immunosuppressive therapy and support the use of annual influenza vaccination in this group of patients, regardless of whether they are receiving immunosuppressive therapy, conclude the researchers. The research team plans to investigate the effect of other vaccines in this group of patients next and hopes to find similarly encouraging outcomes.

Susan J. Allison

Original article Lu, Y. et al. Immune response to influenza vaccine in children with inflammatory bowel disease. Am. J. Gastroenterol. 104, 444-453 (2009). 